\title{
Teratological changes in postembryos of Eratigena atrica obtained by the application of alternating temperatures on spider embryos
}

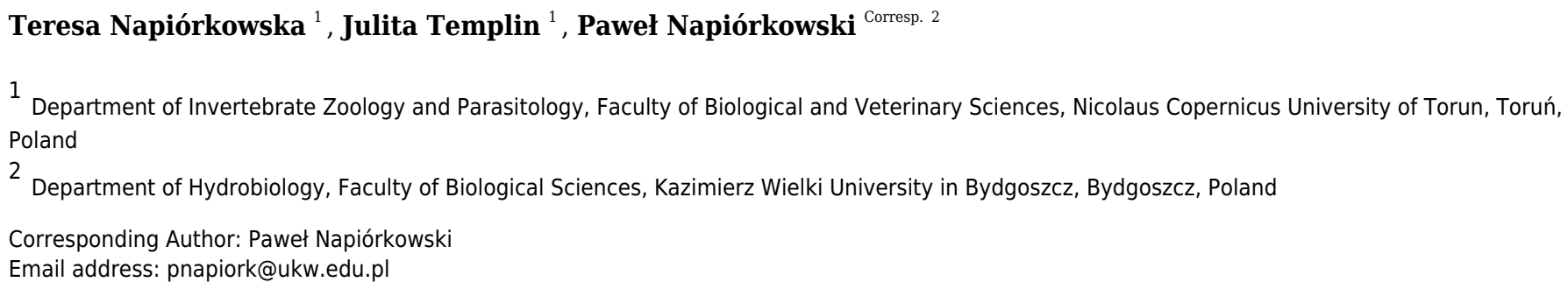

Spider embryonic development depends on several factors, including temperature. Under optimum thermal conditions embryogenesis proceeds undisturbed and embryo mortality is low. On the other hand, dramatic shifts in incubation temperature may cause a range of developmental defects in embryos. It has been confirmed in numerous laboratory experiments that abrupt temperature changes can be a powerful teratogenic factor. Changes in the external structure are frequently reflected in the internal anatomy, and above all, in the central nervous system. In the present teratological study, by exposing spider embryos to the temperatures of $14^{\circ} \mathrm{C}$ and $32^{\circ} \mathrm{C}$, changed every 12 hours for the first 10 days of their development, we obtained 74 postembryos of Eratigena atrica with body deformities such as oligomely, heterosymely, schistomely, bicephaly, complex anomalies and others. We selected six spiders to describe and analyze their morphological changes. In one case, that of a spider affected by polymely (the presence of a supernumerary appendage) combined with heterosymely (the fusion of walking legs), we also focused on the structure of the central nervous system. The analysis indicated that this complex anomaly was accompanied by only one change in the central nervous system: the presence of a supernumerary neuropil. Since no fusion of walking leg neuropils was observed, it was concluded that, in this instance, there was no relationship between the fusion of legs and the structure of the central nervous system. 
1 Teratological changes in postembryos of Eratigena atrica obtained by the application of

2 alternating temperatures on spider embryos

3

4 Teresa Napiórkowska ${ }^{\mathrm{a}}$, Julita Templina ${ }^{\mathrm{a}}$, Paweł Napiórkowski ${ }^{\mathrm{b}}$

$5 \quad$ aDepartment of Invertebrate Zoology and Parasitology, Faculty of Biological and Veterinary

6 Sciences, Nicolaus Copernicus University, Lwowska 1 Street, 87-100 Toruń, Poland

7 bDepartment of Hydrobiology, Faculty of Biological Sciences, Kazimierz Wielki University,

8 Ossollińskich 12 Street, 85-093 Bydgoszcz, Poland

9

10 Corresponding author: Paweł Napiórkowski, Department of Hydrobiology, Faculty of Biological

11 Sciences, Kazimierz Wielki University, Ossollińskich 12 Street, 85-093 Bydgoszcz, Poland

12 e-mail address: pnapiork@ukw.edu.pl 
Abstract

21 Spider embryonic development depends on several factors, including temperature. Under optimum thermal conditions embryogenesis proceeds undisturbed and embryo mortality is low.

On the other hand, dramatic shifts in incubation temperature may cause a range of developmental defects in embryos. It has been confirmed in numerous laboratory experiments that abrupt temperature changes can be a powerful teratogenic factor. Changes in the external structure are frequently reflected in the internal anatomy, and above all, in the central nervous system. In the present teratological study, by exposing spider embryos to the temperatures of $14^{\circ} \mathrm{C}$ and $32^{\circ} \mathrm{C}$, changed every 12 hours for the first 10 days of their development, we obtained 74 postembryos of Eratigena atrica with body deformities such as oligomely, heterosymely, schistomely, bicephaly, complex anomalies and others. We selected six spiders to describe and analyze their morphological changes. In one case, that of a spider affected by polymely (the presence of a supernumerary appendage) combined with heterosymely (the fusion of walking legs), we also focused on the structure of the central nervous system. The analysis indicated that this complex anomaly was accompanied by only one change in the central nervous system: the presence of a supernumerary neuropil. Since no fusion of walking leg neuropils was observed, it was concluded that, in this instance, there was no relationship between the fusion of legs and the structure of the central nervous system. 
Introduction

44 Teratology, a relatively new field of knowledge, only coming into existence in the early 19 th century, studies the causes, mechanisms, and patterns of abnormal development (Ujházy et al., 2012 and references therein). Owing to advances in genetics, toxicology, molecular biology, animal testing, and research on living organisms-environment interactions, teratology has developed significantly in recent years (Calado \& dos Anjos Pires, 2018). Currently there are many known teratogenic factors. Their teratogenicity has been confirmed in numerous experiments, which aid our understanding of both developmental defects and their mechanisms and normal processes occurring during embryogenesis (Wilson, 1964). According to the principles of teratology/developmental toxicology, toxins acting on embryos cause dysmorphogenesis when applied in a sufficient dose during a sensitive period in the development

54 of a sensitive species. Though in vitro studies can provide reliable means to assess the potency of teratogenic/toxic substances, there is still a need to use animal models to demonstrate their embryotoxicity (Carvan III et al., 2004). Invertebrates seem to be particularly useful for testing

57 the toxicity/teratogenicity of different factors, including environmental ones. These animals occupy key positions in the food chain, in aquatic and terrestrial ecosystems, and some species or groups of species are found throughout the entire habitat. They have been used for decades in toxicity tests so they have an enormous potential to help identify environmental hazards. Invertebrates have a number of characteristics that facilitate their breeding including small size, 
62 high fertility rate and short lifespan. These factors, together with low purchasing cost, ensure

63 relatively easy and very efficient application in laboratory testing (Lagadici \& Caquet, 1998).

64

65

66

Arthropods, including spiders, in which the body is divided into the prosoma and opisthosoma, are considered to be excellent models for teratological research. Various teratogenic agents applied to spider embryos may cause deformities in both tagmata. Most commonly, these defects are found on the prosoma and its appendages and are easy to detect. In addition, processing a histological specimen for examination, i.e. for an assessment of changes in the internal structure, is a straightforward task. The synanthropic spider Eratigena atrica (C.L. Koch) (previously Tegenaria atrica) from the family Agelenidae has been widely used in teratology research. Important features of this species are the relatively long breeding season in autumn/winter, high fertility rate and large embryo size. A number of experiments have been carried out to induce developmental deformities in this spider species (Jacuński, 1969; Napiórkowska, Jacuński \& Templin, 2010b; Napiórkowska, Napiórkowski \& Templin, 2016a; Napiórkowska \& Templin, 2017a; Napiórkowska \& Templin, 2017b; Napiórkowska \& Templin, 2018).

Various developmental abnormalities in natural populations of terrestrial and aquatic animals have been documented so far. There is an abundant amount of information on body deformities in many groups of arthropods including crustaceans, insects, myriapods, and chelicerates (e.g. Asiain \& Márquez, 2009; Ćurčić et al., 1991; Estrada-Peña, 2001; Fernandez, Gregati \& Bichuette, 2011; Ferreira, 2011; Feuillassier et al., 2012; Kozel \& Novak, 2013; Leśniewska et al., 2009; Levesque et al., 2018). Since many of these teratogenically modified animals were collected in the natural environment, the causes of their deformities remain unknown. Numerous biological factors (including mutations of the germ or somatic cells) as well as mechanical, physical, and chemical ones can be considered as possible determinants of anomalies in 
85

86

87

88

89

90

91

92

93

94

arthropods (e.g. Miličić, Pavković-Lučić \& Lučić, 2013). Deformities can also be induced in strictly controlled laboratory conditions using certain teratogenic agents, e.g. chemical reagents such as cytochalasin $\mathrm{B}$, dithiothreitol, $\alpha$ - lipoic acid, $\mathrm{NaHCO}_{3}$, manganese, lead (Itow \& Sekiguchi, 1979; Itow \& Sekiguchi, 1980; Köhler et al., 2005; Pinsino et al., 2010) and colchicine (Buczek et al., 2019), radiation such as X-rays (Matranga et al., 2010), and physical parameters such as humidity (Buczek, 2000) and temperature. Holm (1940) was the first to put forward a hypothesis about a teratogenic effect of temperature on spiders. Later, Juberthie (1962) studied the impact of elevated temperature on the embryonic development of harvestmen. In subsequent years laboratory experiments confirmed the thesis that abrupt temperature changes could be a powerful teratogenic factor for Eratigena atrica embryos (Jacuński, 1971; Jacuński, 1984; Jacuński \& Templin 2003; Napiórkowska, Jacuński \& Templin, 2010a; Napiórkowska, Jacuński \& Templin, 2010b; Napiórkowska, Napiórkowski \& Templin, 2016a; Napiórkowska, Napiórkowski \& Templin, 2016b). The application of alternating temperatures (lower and higher than the optimum) during the early stages of embryonic development of $E$. atrica led to a range of deformities of the prosoma and opisthosoma (Jacuński, 1984). Understandably, some of these changes prevented deformed individuals from going through successive stages of postembryogenesis. With seriously impaired locomotion, they were unable to hunt, feed, moult and reproduce. Numerous anomalies, including oligomely (absence of one or more appendages), symely (fusion of appendages of the same pair), schistomely (bifurcation of appendages), heterosymely (fusion of adjacent appendages), polymely (appearance of one or more additional appendages), bicephaly (presence of two heads), and so-called complex anomalies (several anomalies occurring simultaneously), have been identified in teratogenic studies (Jacuński \& Napiórkowska, 2000; Jacuński, Templin \& Napiórkowska, 2005; Napiórkowska \& Templin, 
108 109

110

111

112

113

114

115

116

117

118

119

120

121

122

123

124

125

126

127

128

129

130

2013; Napiórkowska, Jacuński \& Templin, 2007; Napiórkowska, Napiórkowski \& Templin, 2015; Napiórkowska, Templin \& Napiórkowski, 2013). Some of them (oligomely) were observed with high frequency, others were quite rare (bicephaly) (Jacuński, Templin \& Napiórkowska, 2005; Templin, Jacuński \& Napiórkowska, 2009). In many instances, the description of morphological defects was followed by a histological analysis of deformed spiders. Particular attention was paid to the central nervous system (Napiórkowska, Jacuński \& Templin, 2010a; Napiórkowska, Jacuński \& Templin, 2010b; Napiórkowska \& Templin, 2017a; Napiórkowska, Templin \& Wołczuk, 2017).

The structures of the digestive and nervous systems have been extensively analyzed in individuals with complex anomalies. The results indicated different internal effects depending on the anomaly (Napiórkowska, Napiórkowski \& Templin, 2015; Napiórkowska, Templin \& Wołczuk, 2017): morphological deformities were not always reflected in the internal anatomy. Therefore, preparation of histology slides of individuals affected by new types of complex anomalies would facilitate the classification of the defects.

In the breeding season $2017 / 2018$ the application of alternating temperatures during early embryogenesis of Eratigena atrica provided us with new, interesting cases in the teratogenic material. Although this method has been used in teratology research on this spider species for years, it can still produce unpredictable results. These new, random anomalies are worth discussing in detail. Our study also emphasizes the power of temperature as a teratogenic agent, whose application in the laboratory may cause such extensive changes in spider anatomy and morphology that affected individuals are unable to express normal behaviour or develop a reproductive strategy. Therefore, the aim of the study was to show the diversity of anomalies in terms of morphological changes as well as, in one spider, anatomical changes. In the latter case it 
131 was hypothesized that morphological changes were reflected in the structure of the central

132 nervous system.

134 Material and methods

135 The teratological experiment involved embryos of Eratigena atrica (C.L.Koch, 1843). 24

136 sexually mature females and 17 males were collected in early autumn near the towns of Chełmża

137 and Torun (Poland) and transported to the laboratory, where each spider was put into a separate

138 glass container with a capacity of $250 \mathrm{~cm}^{3}$. Spiders were kept in a dark room at the temperature

139 of $21-23^{\circ} \mathrm{C}$ and relative humidity $(\mathrm{RH})$ of $70 \%$. Three weeks after the culture was established,

140 males were introduced into containers with females ready for insemination. First egg sacs were

141 laid after several weeks. Embryos were removed from each egg sac, counted, and divided

142 equally into two groups: experimental and control. The control group was kept at the temperature

143 of $22^{\circ} \mathrm{C}$ and $70 \% \mathrm{RH}$, while the experimental group was exposed to temperatures of $14^{\circ} \mathrm{C}$ and

$14432^{\circ} \mathrm{C}(70 \% \mathrm{RH})$ applied alternately every 12 hours. The procedure continued for ten days, until

145 first metameres of the prosoma appeared on the germ band and the leg formation process began

146 (embryo development was observed in paraffin oil; the chorion becomes transparent in paraffin

147 oil). Subsequently, all experimental embryos were incubated under the same conditions as the

148 control ones. After hatching, all control and experimental postembryos (by Downes, 1987) were

149 examined for developmental deformities on the prosoma and opisthosoma. Deformed

150 individuals were photographed using a light microscope (Axcio Lab A1). Images were recorded

151 using a digital camera (Axiocam 105 color, Carl Zeiss) and a computer system running Zen

152 software (Version 2.3, blue edition). One individual was subjected to histological analysis, in

153 which 7- $\mu \mathrm{m}$-thick paraffin sections were stained with Mayer hematoxylin and eosin (Mayer's 
154 haemalum technique). The same method was used to make histological preparations of one

155 individual from the control group.

156 Results

157 In the breeding season 2017/18 we obtained approximately 6000 embryos, half of which

158 constituted the control group. Individuals from this group were not affected by any

159 developmental defects and the mortality rate was low (8\%). They had six pairs of normally

160 developed prosomal appendages and histological analysis of one individual showed no

161 aberrations in the central nervous system (Figure 1).

162 In contrast, in the experimental group the mortality rate of embryos was much higher (37\%).

163 This group contained several postembryos with teratogenic changes on the prosoma or

164 opisthosoma. In total, 74 out of 1,900 postembryos were affected by one of the following

165 anomalies within the prosoma: oligomely, heterosymely, schistomely, bicephaly, complex

166 anomaly and others. In the latter group were postembryos with considerably shorter appendages

167 of the prosoma, protuberances of different size and shape or anomalies in the spinning apparatus

168 (Table 1). Several interesting cases selected for analysis are presented in Figure 2.

169 The spider in Figure 2A was affected by bilateral oligomely and, apart from one pair of

170 pedipalps (P), had only three pairs of walking legs (L1-L3). Additionally, it had a truncheon-

171 shaped protuberance (A) in place of the right chelicera (ventral view). A similar protuberance

172 (A) developed in place of one pedipalp in the spider presented in Figure 2B. The remaining

173 appendages on the prosoma, i.e. chelicerae (C), left pedipalp (P) (dorsal view) and walking legs

174 (L1-L4) were well-developed and had the correct size and segmentation. In the spider in Figure

$1752 \mathrm{C}$ a complex anomaly was observed. The specimen had one chelicera (C), one pedipalp (P) and 
176 only two walking legs (L1 and L2) on the right side of the prosoma (ventral view). The left side

177 of the prosoma was significantly changed. Behind the chelicera (C) there was a schistomelic

178 pedipalp (P), with one free end much shorter and deformed. Behind the pedipalp there was also a

179 short protuberance (A), widened in the middle, and only two walking legs (L1 and L2). A

180 complex anomaly was also recognized in the spider in Figure 2D. This individual was affected

181 by oligomely of walking legs on the right side of the prosoma (ventral view), because behind the

182 fully formed chelicera (C) and pedipalp (P) there were only three walking legs (L1-L3). On the

183 left side of the prosoma the first walking leg (L1) was schistomelic. The bifurcation started at the 184 tibia. The non-bifurcated part of the leg was thicker than usual, with distinct segmentation. The

two free ends, which extended in opposite directions, were also distinctly segmented. Posterior to 186 the schistomelic leg were three well-developed walking legs (L2-L4). Figures 2E and 2F show 187 two different bicephalous specimens. One (Fig. 2E) had two equivalent heads with a double set 188 of chelicerae $(\mathrm{C})$ and pedipalps $(\mathrm{P})$. Between the heads were two fully formed, separate walking 189 legs (L). The other specimen (Fig. 2F) had two complete heads with chelicerae (C), pedipalps (P) 190 and two walking legs (L/L) in between, fused from coxa to the patella. Both individuals also had 191 a standard set of walking legs (L1-L4).

192 A histological analysis was performed on one individual affected by an anomaly that had not 193 been previously recorded. The case seemed interesting both in terms of morphology and internal 194 anatomy. As can be seen in Figure 3A the spider had a complex anomaly, i.e. polymely and 195 heterosymely of the walking legs on the right side of the prosoma (dorsal view). Behind a well196 formed, six-segmented pedipalp (P) was a very thick, significantly deformed appendage (a) with 197 two free ends (L1; L2A/2B). Based on its unique appearance, it was assumed that it consisted of 198 three walking legs, which would mean that five walking legs developed on this side of the 
199 prosoma (polymely). The last two legs (L3; L4) were well-developed with seven segments. The

200 first three were heterosymelic. Two of them (L2A/2B) were fused over their entire length (total

201 heterosymely). In addition, they were fused with the first leg (L1) along the coxae, trochanters,

202 femurs, and patellas (partial heterosymely), which explains the presence of the two free distal

203 ends: L1 and L2A/2B. The assumption was that the end L2A/2B was much thicker because it

204 was composed of the last three segments of the completely fused legs. The segmentation of this

205 end was very indistinct, but its length was the same as that of the end of L1, which consisted of

206 three segments. On the opposite side of the prosoma there was a set of properly formed

207 appendages.

208 To verify our assumptions we prepared histology slides of the central nervous system of the

209 investigated spider. There were no structural changes in the brain (Fig. 3B). However, in the

210 ventral nerve cord (subesophageal ganglia) the number of leg neuropils was higher. Figure 3C

211 shows the neuropils (n) of the ventral nerve cord in its middle part, with four separate walking

212 leg neuropils (n1, n2A, n3, n4) in addition to the neuropil of the pedipalp (np) on the deformed

213 right side. The last two on this side of the prosoma $(n 3, n 4)$ were the neuropils of the well-

214 formed walking legs L3 and L4: the first two (n1, n2A), those of the heterosymelic legs. Based

215 on the location, n1 was assumed to be the neuropil of the leg whose distal end was marked as L1

216 and n2A was neuropil for one of the totally fused L2A/2B (end L2A/2B). The ventral nerve cord

217 contained one abnormal additional neuropil, displaced ventrally (n2B) (Fig. 3D). Histological

218 analysis indicated that it belonged to the second leg of the fused complex, whose end was

219 marked as L2A/2B. No fusion of the leg neuropils was observed.

Discussion 
222 For the present study, 1,900 postembryos that left their eggshells after exposure to the

223 teratogenic agent (alternating sub- and supra-optimal temperatures) were examined for

224 developmental deformities. 74 individuals, i.e. 3.9\%, had body defects. Assuming that thermal

225 shocks applied during spider embryogenesis are a potent teratogen, the number seems low. There

226 may be multiple possible reasons of such a low frequency of deformities.

227 Firstly, it may be connected with expression of heat shock protein (Hsp) genes. In general, Hsp

228 genes are expressed at low levels under normal growing conditions, but their expression

229 increases considerably in response to different environmental stressors such as heat, desiccation

230 or heavy metals. Hsp genes represent a subset of a larger group of genes coding for molecular

231 chaperones. Chaperones can assist in the efficient folding of nascent peptides, also acting when

232 denatured proteins accumulate in cells, preventing them from irreversible aggregation and

233 misfolding (Martinez-Paz et al., 2014 and references therein). Secondly, effective repair

234 processes at every stage of embryo development may eliminate errors that occur during

235 morphogenesis. Finally, spiders, which are ectothermic animals, must be relatively resistant to

236 abrupt temperature changes. This would also apply to spider embryos, although their mortality

237 was relatively high $(37 \%)$.

238 Oligomelic individuals were the most numerous in the teratological material (over 50\%), which

239 coincides with the results of previous studies (Jacuński, 1984; Napiórkowska, Napiórkowski \&

240 Templin, 2016b). A relatively large group of postembryos $(23 \%)$ had deformities classified as

241 'Others' in Table 1, followed by postembryos with so-called complex anomalies (15\%). Since

242 many deformities obtained by the application of temperature changes during embryonic

243 development of Eratigena atrica have already been described (e.g. Napiórkowska, Napiórkowski

244 \& Templin, 2015; Napiórkowska, Napiórkowski \& Templin, 2016a), we focused on those that 
245 were encountered for the first time. Based on the previous observations it can be predicted that

246 new surprising changes may occur when developmental processes are disturbed by temperature

247 shocks. Every year novel body deformities are registered in teratological experiments.

248 In one case we analyzed not only deformities of the walking legs on the right side of the prosoma

249 but also the structure of the central nervous system. The nature of this malformation suggests two

250 processes: the formation of an additional leg (polymely) and the fusion of three walking legs

251 (heterosymely). Only the polymely was reflected in the central nervous system: an additional

252 walking leg neuropil was found in the ventral nerve cord, but neuropil fusion was not observed.

253 Therefore, on the right side of the ventral nerve cord there were five walking leg neuropils and

254 one of them was shifted to the ventral side. According to Jacuński (1984), an additional leg, not

255 developed during normal ontogenesis, is associated with the appearance of an additional half of a

256 metamere (and thus of a neuromere) on the germ band. This suggests that all polymelic legs

257 should have their ganglia, as has been observed in numerous studies (e.g. Napiórkowska,

258 Napiórkowski \& Templin, 2015). However, two different scenarios have been observed in

259 instances of leg polymely: 1) an increased number of ganglia and their fusion, despite the

260 absence of fused legs (Napiórkowska, Napiórkowski \& Templin, 2015; Napiórkowska, Templin

$261 \&$ Wołczuk, 2017), 2) an increased number of ganglia and no leg or ganglia fusions (Jacuński et

262 al., 2002; Napiórkowska, Jacuński \& Templin, 2006).

263 Based only on the morphology of the spider in Fig. 3, another possibility could be considered,

264 namely that the fused walking legs consist of only two legs (despite relatively big difference in

265 thickness of the two free ends). In teratological studies, leg deformities consisting of significant

266 thickening, narrowing or curving, which are frequently observed in postembryos, disappear after

267 several molts (personal observation). Therefore, the thicker end (L2A/2B) could, in theory, 
268 belong to one leg. However, histological analysis indicated the presence of an additional,

269 polymelic leg, whose neuropil (n2B) was shifted to the ventral side. Additionally, intervening

270 sections did not reveal continuity between $\mathrm{n} 2 \mathrm{~A}$ and $\mathrm{n} 2 \mathrm{~B}$. For that reason it can be concluded that

271 neuropil $\mathrm{n} 2 \mathrm{~B}$ is not part of a distorted n2A but of the additional leg. However, even if such a

272 continuity had been discovered, not only complete heterosymely of the legs (L2A/2B), but also

273 of the corresponding neuropiles (n2A and n2B) could have been a possible diagnosis.

274 The spatial location of the ganglia is another issue. In the majority of cases the ganglia

275 (neuropils), including the supernumerary ones, were located in one plane (Napiórkowska,

276 Templin \& Wołczuk, 2017). In several individuals the ganglia were shifted to the dorsal or

277 ventral side (Napiórkowska, Napiórkowski \& Templin, 2015). It is therefore important to

278 understand the causes of these shifts. First, they may be induced by changes in the genes

279 responsible for the formation of the anterior-posterior and dorsal-ventral axes, which determine

280 the location of all internal organs and structures. Based on latest reports (e.g. Damen et al., 1998;

281 Khadjech et al., 2012; Pechmann et al., 2009; Schwager et al., 2009; Telford \& Thomas, 1998)

282 we suppose that these shifts resulted from changes in Hox gene expression patterns. Hox genes

283 are able to alter the arthropods plan, as well as, determine the presence or absence of legs in

284 different parts of the body (e.g. Antennapedia and Distal-less genes). Another explanation could

285 be space limitation: since the size and symmetry of the prosoma does not change (despite the

286 presence of an additional leg and additional half of a neuromere) an additional ganglion has to be

287 "pushed", ventrally or dorsally in order to fit into a limited volume of the prosoma.

288 In the investigated spider the heterosymely of walking legs was not associated with the fusion of

289 their ganglia (neuropils), although it seems logical that it could have been reflected in the central

290 nervous system. This would have suggested a certain hierarchy between segmental structures, 
291 with the fusion of ganglia leading to the fusion of the corresponding legs. Such a situation was

292 observed in bicephalous E. atrica whose chelicerae and pedipalps were completely fused

293 (Napiórkowska et al., 2016c). However, in the vast majority of cases, heterosymely has not been

294 accompanied by the fusion of leg ganglia (Napiórkowska, Napiórkowski \& Templin, 2015;

295 Napiórkowska, Templin \& Napiórkowski, 2013). This indicates that the fusion of walking legs

296 may result from the fusion of the developing leg buds, caused by the exposure of an early

297 embryo to thermal shock. In other words, thermal shock may affect the developing leg buds, but

298 not necessarily leg ganglia. Temperature changes applied during embryo incubation may or may

299 not affect various elements of serial structures, such as ganglia or legs. The consequences depend

300 on the intensity of the thermal shock and time of its application. From this point of view, an

301 application of a teratogen in early stages of embryogenesis may cause more profound changes

302 than its later application and a range of effects may be expected. Moreover, since morphological

303 defects are not always reflected in the central nervous system (an example of which is the

304 investigated E. atrica), teratological studies should not be limited to deformity descriptions but

305 should also focus on internal anatomical examination, including that of the central nervous

306 system. This type of research has already been conducted on spiders and other arthropods

307 (Harzsch, Benton \& Beltz, 2000; Jacuński, Templin \& Napiórkowska, 2005; Scholtz, Ng \&

308 Moore, 2014).

309 A certain analogy can be seen between the investigated E. atrica and a deformed pycnogonid

310 Pycnogonum litorale described by Scholtz \& Brenneis (2016). In the latter, an extensive

311 deformity resulted from a mechanical, unintentional injury to the region between the second and

312 third walking leg. After several months the sea spider developed an extra leg on the right side of

313 the prosoma partially fused with other legs. In this case, the supernumerary leg did not have an 
314 associated ganglion although it did, like the other legs, contain a midgut diverticulum and a

315 branch of ovary. Scholtz \& Brenneis (2016) explained the anomaly using the "boundary model"

316 proposed by Meinhardt in the 1980s and supported later by molecular data. This model

317 hypothesized the division of each body segment into at least three cellular compartments,

318 designated S, A and P, along the antero-posterior axis (Meinhardt, 1986). Two of these were

319 known from Drosophila research, with each segment comprised of transverse cell populations

320 with an anterior or a posterior fate, the A and P compartments, respectively, which lie strictly

321 separated but adjacent to each other (Martinez-Arias \& Lawrence, 1985). Meinhardt' model

322 further hypothesized that, perpendicular to each A-P border, there is a longitudinal boundary

323 separating dorsal (D) and ventral (V) cells on either lateral side of the embryo (Meinhardt,

324 1986). At intersections between A-P and D-V borders, the formation of limb buds is initiated. If

325 the cells of an S compartment are removed, the P cells of an anterior segment form a contact

326 zone with the A cells of the more posterior segment and an additional leg is formed. This model

327 could also be used to explain the formation of an additional leg in the investigated E. atrica.

328 Furthermore, molecular analysis might help explain the mechanisms of morphological defects in

329 this spider. Many researchers, including Khadjeh et al. (2012) and Pechmann et al. (2011) have

330 successfully conducted such studies on spiders.

331 All developmental defects, both those that are caused by some complex regenerative processes

332 and those that are caused by teratogenic factors (e.g. alternating temperatures applied during

333 early embryogenesis), can contribute to a better understanding of spider phylogenesis and

334 development. For example, the abnormal development of a short appendage on the petiolus

335 (pedicel) in a postembryo of E. atrica, as observed by Jacunski and Templin (1991), has been

336 interpreted as an atavistic feature which might facilitate determining how spiders are related to 
337 other groups of arthropods. Obviously, this issue would require in-depth research. In addition,

338 developmental defects indicate morphological capabilities and developmental potential of

339 organisms, not displayed under normal conditions.

340 Teratological experiments based on temperature seem justified in view of current weather

341 anomalies. Sudden temperature changes observed nowadays can affect embryos, causing damage

342 that may not be subject to spontaneous repair processes. As a consequence, a higher number of

343 deformed animals may be found in the natural environment. Observation of spiders, commonly

344 found near human settlements, can provide abundant evidence of adverse environmental impacts.

345 In conclusion, our results suggest that temperature changes during embryonic development of

346 animals can cause various deformities in their body structure. Our findings provide additional

347 evidence that morphological defects are not always reflected in the central nervous system (an

348 example of which is the investigated E. atrica). Therefore, teratological studies should not be

349 limited to describing external features of deformed individuals, but should also involve

350 analyzing their internal organs, including the CNS.

352 Declaration of competing interest

353 The authors declare they have no known competing financial interests or personal relationships

354 that could have appeared to influence the work reported in this paper.

355 Acknowledgements

356 This work was supported by the Faculty of Biological and Veterinary Sciences of the Nicolaus

357 Copernicus University in Torun [statutory fund research] and Faculty of Biological Sciences of

358 the Kazimierz Wielki University in Bydgoszcz (Poland). 
360

361

362

363

364

365

366

367

368

369

370

371

372

373

374

375

376

377

378

379

380

381

382

References

Asiain J, Márquez J. 2009. New teratological examples in neotropical Staphylinidae (Insecta:

Coleoptera), with a compilation of previous teratological records. Revista Mexicana de

Biodiversidad 80: 129-139.

Calado AM, dos Anjos Pires M. 2018. An Overview of Teratology. In: Félix L, ed.

Teratogenicity Testing. Methods in Molecular Biology. Humana Press, New York, 3-32.

https://doi.org/10.1007/978-1-4939-7883-0 1

Buczek A. 2000. Experimental teratogeny in the tick Hyalomma marginatum marginatum

(Acari: Ixodida: Ixodidae): effect of high humidity on embryonic development. Journal of

Medical Entomology 37: 807-814. https://doi.org/10.1603/0022-2585-37.6.807

Buczek A, Bartosik K, Buczek AM, Buczek W, Kulina D. 2019. Abnormal development of

Hyalomma marginatum ticks (Acari: Ixodidae) induced by plant cytotoxic substances. Toxins

11(8): 445. https://doi.org/10.3390/toxins11080445

Carvan III MJ, Loucksa E, Weberb DN, Williams FE. 2004. Ethanol effects on the developing zebrafish: neurobehavior and skeletal morphogenesis. Neurotoxicology and Teratology 26:

757-768. https://doi.org/10.1016/j.ntt.2004.06.016

Ćurčić BPM, Dimitrijević RN, Karamata OS, Lučić LR. 1991. Segmental anomalies in Roncus aff. lubricus (Neobisiidae, Pseudoscorpiones) from Yugosłavia. The Journal of Arachnology 19: 215-224.

Damen WGM, Hausdorf M, Seyfarth E-A, Tautz D. 1998. A conserved mode of head segmentation in arthropods revealed by the expression pattern of Hox genes in a spider.

Proceedings of the National Academy of Sciences of the United States of America 95: 1066510670. https://doi.org/10.1073/pnas.95.18.10665 
383 Downes MF. 1987. A proposal for standardization of the terms used to describe the early

384 development of spiders, based on a study of Theridion rufipes Lucas (Araneae: Theridiidae).

385 Bulletin of the British arachnological Society 7: 187-193.

386 Estrada-Peña A. 2001. Abnormal development of Rhipicephalus sanguineus (Ixodidae).

387 Experimental and Applied Acarology 25: 757-761. https://doi.org/10.1023/A:1016310214918

388 Fernandez CS, Gregati RA, Bichuette ME. 2011. The first record of external abnormalities in the 389 subterranean Aegla marginate Bond-Buckup \& Buckup, 1994 (Crustacea: Decapoda: Aeglidae), 390 from a karst area of Southnearsten Brasil. Subterranean Biology 8: 33-38.

391 https://doi.org/10.3897/subtbiol.8.1228

392 Ferreira RN. 2011. Three anomalies of Coleoptera (Carabidae, Staphylinidae, and Scarabaeidae)

393 from Connecticut. Insecta Mundi 0169: 1-3.

394 Feuillassier L, Beuger M, Pauliac G, Boët P, Girardin M, Elie P. 2012. Morphological anomalies

395 in estuarine shrimp larvae. Crustaceana 85 (1): 11-25.

396 https://doi.org/10.1163/156854012X623647

397 Harzsch S, Benton J, Beltz BS. 2000. An unusual case of a mutant lobster embryo with double

398 ventral nerve cord. Arthropod Structure and Development 29: 95-99.

399 https://doi.org/10.1016/S1467-8039(00)00016-5

400 Holm A. 1940. Studien über die Entwicklungsbiologie der Spinen. Zoologische Beiträge aus

401 Uppsala 19: 1-214. [in German].

402 Itow T, Sekiguchi K. 1979. Induction of multiple embryos with $\mathrm{NaHCO}_{3}$, or calcium free sea

403 water in the horseshoe crab. Wilhelm Roux's archives of developmental biology 187: 245-254.

404 https://doi.org/10.1007/BF00848620 
405 Itow T, Sekiguchi K. 1980. Morphogenic movement and experimentally induced decrease in 406 number of embryonic segments in the Japanese horseshoe crab, Tachypelus tridentatus. Biology 407 Bulletin 158 (3): 324-338. https://doi.org/10.2307/1540859

408 Jacuński J. 1969. Inducement of developmental monstrosities in the spider Tegenaria atrica 409 C.L. Koch by centrifugation of mature females. Zoologica Poloniae 19(4): 589-600.

410 Jacuński L. 1971. Temperature induced developmental monstrosities in Tegenaria atrica C. L. 411 Koch (Araneae, Agelenidae). Zoologica Poloniae 21: 285-316.

412 Jacuński L. 1984. Studies on experimental teratogeny in the spider Tegenaria atrica C. L. Koch. 413 D. Phil. Thesis, Nicolaus Copernicus Press Toruń, Poland. [in Polish].

414 Jacuński L, Napiórkowska T. 2000. Epimorphic regeneration of an appendage complex in 415 Tegenaria atrica C. L. Koch (Agelenidae). Bulletin of the Polish Academy of Sciences, 416 Biological Sciences 48: 269-271.

417 Jacuński L, Napiórkowska T, Templin J, Tesznar L. 2002. Interesting cases of polymely in 418 Tegenaria atrica C. L. Koch (Agelenidae). Bulletin of the Polish Academy of Sciences, 419 Biological Sciences 50(2): 149-151.

420 Jacuński L, Templin J. 1991. Leg on the petiolus of a spider - Tegenaria atrica C. L. Koch in the 421 postembryonic development. Przeglad Zoologiczny XXXV (1-2): 135-138. [in Polish].

422 Jacuński L, Templin J. 2003. Morphology of prosoma in bicephalous monster of Tegenaria 423 atrica. C. L. Koch. Journal of Thermal Biology 28: 393-396. https://doi.org/10.1016/S0306$424 \quad \underline{4565(03) 00023-8}$

425 Jacuński L, Templin J, Napiórkowska T. 2005. Changes in the neuromerism of the 426 subesophageal part of the nervous system in oligomelic individuals of Tegenaria atrica 427 (Arachnida). Biologia, Bratislava 60 (5): 589-592. 
428 Juberthie C. 1962. Etude des symélies provoquées par la temperature chez un Opilions

429 (Arachnides). Comptes Rendus de l'Académie des Sciences 254: 2674-2676.

430 Khadjeh S, Turetzek N, Pechmann M, Schwager EE, Wimmer EA, Damen WGM, Prpic N-M.

431 2012. Divergent role of the Hox gene Antennapedia in spiders is responsible for the convergent

432 evolution of abdominal limb repression. Proceedings of the National Academy of Sciences of the

433 United States of America 109: 4921-4926. https://doi.org/10.1073/pnas.1116421109

434 Kozel P, Novak T. 2013. Absence of a ventral spur on the chelicerae in Lacinius ephippiatus

435 (Oligolophinae: Phalangidae: Opiliones). Entomology News 123 (3): 201-205.

436 https://doi.org/10.3157/021.123.0306

437 Köhler HR, Alberti G, Seniczak S, Seniczak A. 2005. Lead-induced hsp70 and hsp60 pattern

438 transformation and leg malformation during postembryonic development in the oribatid

439 mite, Archegozetes longisetosus Aoki. Comparative Biochemistry and Physiology Part C:

440 Toxicology and Pharmacology 141 (4): 398-405. https://doi.org/10.1016/j.cbpc.2005.09.003

441 Lagadici L, Caquet T. 1998. Invertebrates in Testing of Environmental Chemicals: Are They

442 Alternatives? Environmental Health Perspectives 106 (Suppl 2): 593-611.

443 https://doi.org/10.1289/ehp.98106593

444 Leśniewska M, Bonato L, Minelli A, Fusco G. 2009. Trunk abnormalities in the centripede

445 Stigmatogaster subterranea give insight into late-embryonic segmentation. Arthropod Structure

446 and Development 38: 417-426. https://doi.org/10.1016/j.asd.2009.05.001

447 Levesque B, Cachot J, Boët P, Lepage M, Mazella N, Martin C, Gourves PY, Legeay A. 2018.

448 Seasonal variations of contamination and exoskeletal malformations in the white shrimps

449 Palaemon longirostris in the Gironde estuary, France. Environmental Science and Pollution

450 Research 25(23). https://doi.org/10.1007/s11356-018-2241-6 
451 Martinez-Arias A, Lawrence PA. 1985. Parasegments and compartments in the Drosophila

452 embryo. Nature 313: 639-642. https://doi.org/10.1038/313639a0

453 Martínez-Paz P, Morales M, Martín R, Martínez-Guitarte JL, Morcillo G. 2014. Characterization

454 of the small heat shock protein Hsp27 gene in Chironomus riparius (Diptera) and its expression

455 profile in response to temperature changes and xenobiotic exposures. Cell Stress and

456 Chaperones 19: 529-540. https://doi.org/10.1007/s12192-013-0479-y

457 Matranga V, Zito F, Costa C, Bonaventura R, Giarrusso S, Celi F. 2010. Embryonic

458 development and skeletogenic gene expression affected by X-rays in the Mediterranean sea

459 urchin Paracentrotus lividus. Ecotoxycology 19: 530-537. https://doi.org/10.1007/s 10646-009-

$460 \quad \underline{0444-9}$

461 Meinhardt H. 1986. The threefold subdivision of segments and the initiation of legs and wings in

462 insects. Trends in Genetics 2: 36-41. https://doi.org/10.1016/0168-9525(86)90173-3

463 Miličić D, Pavković-Lučić S, Lučić L. 2013. On some morphological abnormalities in adult fairy

464 shrimp Branchipus schaeferi Fisher, 1934, from Serbia. Archives of Biological Sciences 65(4):

465 1645-1650. https://doi.org/10.2298/ABS1304645M

466 Napiórkowska T, Jacuński L, Templin J. 2006. The effect of amputation of polymelic walking

467 legs and of parts of appendage complexes in Tegenaria atrica (Araneae: Agelenidae). Bulletin of

468 the British arachnological Society (Arachnology) 13(9): 347-352

469 Napiórkowska T, Jacuński L, Templin J. 2007. Epimorphosis and repair processes of

470 schistomelic pedipalps and walking appendages in Tegenaria atrica (Araneae, Agelenidae).

471 Biologia, Bratislava 62/6: 756-762. https://doi.org/10.2478/s11756-007-0138-9 
472 Napiórkowska T, Jacuński L, Templin J. 2010a. An interesting case of bicephalous Tegenaria

473 atrica nymph. Bulletin of the British arachnological Society (Arachnology) 15: 83-84.

474 https://doi.org/10.13156/arac.2010.15.3.83

475 Napiórkowska T, Jacuński L, Templin J. 2010b. Polymely of feeding appendages in Tegenaria 476 atrica (Araneae, Agelenidae). Bulletin of the British arachnological Society (Arachnology) 15:

477 52-54. https://doi.org/10.13156/arac.2010.15.2.52

478 Napiórkowska T, Napiórkowski P, Templin J. 2015. Morphological and anatomical changes

479 related to leg anomalies in Tegenaria atrica. Zoomorphology 134: 237-245.

480 https://doi.org/10.1007/s00435-015-0260-0

481 Napiórkowska T, Napiórkowski P, Templin J. 2016a. Teratological deformities of pedipalps in 482 the Tegenaria atrica spider, induced by low and high temperatures applied alternately. Journal 483 of Thermal Biology 56: 50-54. https://doi.org/10.1016/j.jtherbio.2015.12.005

484 Napiórkowska T, Napiórkowski P, Templin J. 2016b. Morphological changes of the central 485 nervous system of oligomelic Tegenaria atrica spiders. Folia Biologica Krakow 64 (2): 113-118.

486 https://doi.org/10.3409/fb64_2.113

487 Napiórkowska T, Napiórkowski P, Templin J, Wołczuk K. 2016c. Bicephality, a seldom 488 occurring developmental deformity in Tegenaria atrica caused by alternating temperatures.

489 Journal of Thermal Biology 60: 125-131. https://doi.org/10.1016/j.jtherbio.2016.06.015

490 Napiórkowska T, Templin J. 2013. Symely, a seldom occurring developmental anomaly in the 491 spider Tegenaria atrica. Invertebrate Reproduction and Development 57(2): 95-100.

492 https://doi.org/10.1080/07924259.2012.678391

493 Napiórkowska T, Templin J. 2017a. Bicephality in Eratigena atrica larva. Pakistan Journal of 494 Zoology 49(6): 2339-2341. https://doi.org/10.17582/journal.pjz/2017.49.6.sc6 
495 Napiórkowska T, Templin J. 2017b. Teratological changes on the prosoma of Eratigena atrica

496 spiders caused by alternating temperatures. Invertebrate Survival Journal 14: 480-487.

497 https://doi.org/10.25431/1824-307X/isj.v14i1.480-487

498 Napiórkowska T, Templin J. 2018. Heterosymely and accompanying anomalies in the spider

499 Eratigena atrica (C.L.Koch, 1984) (Araneae, Agelenidae). Annales Zoologici 68(4): 909-914.

500 https://doi.org/10.3161/00034541ANZ2018.68.4.012

501 Napiórkowska T, Templin J, Napiórkowski P. 2013. The central nervous system of

502 heterosymelic individuals of the spider Tegenaria atrica. Folia Biologica (Krakow) 61(3-4):

503 283-289. https://doi.org/10.3409/fb61_3-4.283

504 Napiórkowska T, Templin J, Wołczuk K. 2017. Morphology and the central nervous system of

505 Eratigena atrica affected by a complex anomaly in the anterior part of the prosoma. Invertebrate

506 Neuroscience 17: 11. https://doi.org/10.1007/s10158-017-0204-0

507 Pechmann M, Khadjeh S, Turetzek N, McGregor AP, Damen WGM, Prpic N-M. 2011. Novel

508 function of Distal-less as a gap gene during spider segmentation. PLoS Genetics 7: 1-10.

509 https://doi.org/10.1371/journal.pgen.1002342

510 Pechmann M, McGregor AP, Schwager EE, Feitosa NM, Damen WGM. 2009. Dynamic gene

511 expression is required for anterior regionalization in a spider. Proceedings of the National

512 Academy of Sciences of the United States of America 106: 1468-1472.

513 https://doi.org/10.1073/pnas.0811150106

514 Pinsino A, Matranga V, Trinchella F, Roccheri. 2010. Sea urchin embryos as an in vivo model

515 for the assessment of manganese toxicity: developmental and stress response effects.

516 Ecotoxicology 19: 555-562. https://doi.org/10.1007/s10646-009-0432-0 
517 Scholtz G, Brenneis G. 2016. The pattern of a specimen of Pycnogonum litorale (Arthropoda,

518 Pycnogonida) with a supernumerary leg can be explained with the ,boundary model” of

519 appendage formation. The Science of Nature 103: 13. https://doi.org/10.1007/s00114-016-1333-8

520 Scholtz G, Ng PKL, Moore S. 2014. A crab with three eyes, two rostra, and a dorsal antenna-like

521 structure. Arthropod Structure and Development 43: 165-173.

522 https://doi.org/10.1016/j.asd.2013.10.007

523 Schwager EE, Pechmann M, Feitosa NM, McGregor AP, Damen WGM. 2009. hunchback

524 functions as a segmentation gene in the spider Achaearanea tepidariorum. Current Biology 19:

525 1333-1340. https://doi.org/10.1016/j.cub.2009.06.061

526 Telford MJ, Thomas RH. 1998. Expression of homeobox genes shows chelicerate arthropods

527 retain their deutocerebral segment. Proceedings of the National Academy of Sciences of the

528 United States of America 95: 10671-10675. https://doi.org/10.1073/pnas.95.18.10671

529 Templin J, Jacuński L, Napiórkowska T. 2009. Disturbances in the structure of the prosoma in

530 Tegenaria atrica induced by alternating temperatures (Araneae: Agelenidae). Bulletin of the

531 British arachnological Society 14: 303-307. https://doi.org/10.1016/0002-9378(64)90840-3

532 Ujházy E, Mach M, Navarová J, Brucknerová I, Dubovický M. 2012. Teratology - past, present

533 and future. Interdisciplinary Toxicology 5(4): 163-168. https://doi.org/10.2478/v10102-012-

$534 \underline{0027-0}$

535 Wilson JG. 1964. Experimental teratology. American Journal of Obstetrics and Gynecology 90

536 (7, 2): 1181-1193. https://doi.org/10.1016/0002-9378(64)90840-3

537

538 


\section{Table $\mathbf{1}$ (on next page)}

Types and frequency of anomalies on the prosoma in Eratigena atrica postembryos. 


\begin{tabular}{lcc}
\hline Kind of anomaly & Number of individuals & $\%$ \\
\hline Oligomely & 39 & 52.70 \\
Heterosymely & 3 & 4.06 \\
Schistomely & 1 & 1.35 \\
Bicephaly & 3 & 4.06 \\
Complex anomalies & 11 & 14.86 \\
Others & 17 & 22.97 \\
\hline Total & 74 & 100.00 \\
\hline
\end{tabular}

2 


\section{Figure 1}

Eratigena atrica postembryo from control group.

A ventral view: C, chelicerae; P, pedipalps, L1 - L4, walking legs; B-D horizontal sections through the prosoma, brain (B) and ventral nerve cord $(\mathbf{C}, \mathbf{D})$ : n, neuropil; n1-n4, neuropils of walking legs; $n p$, neuropils of pedipalps.

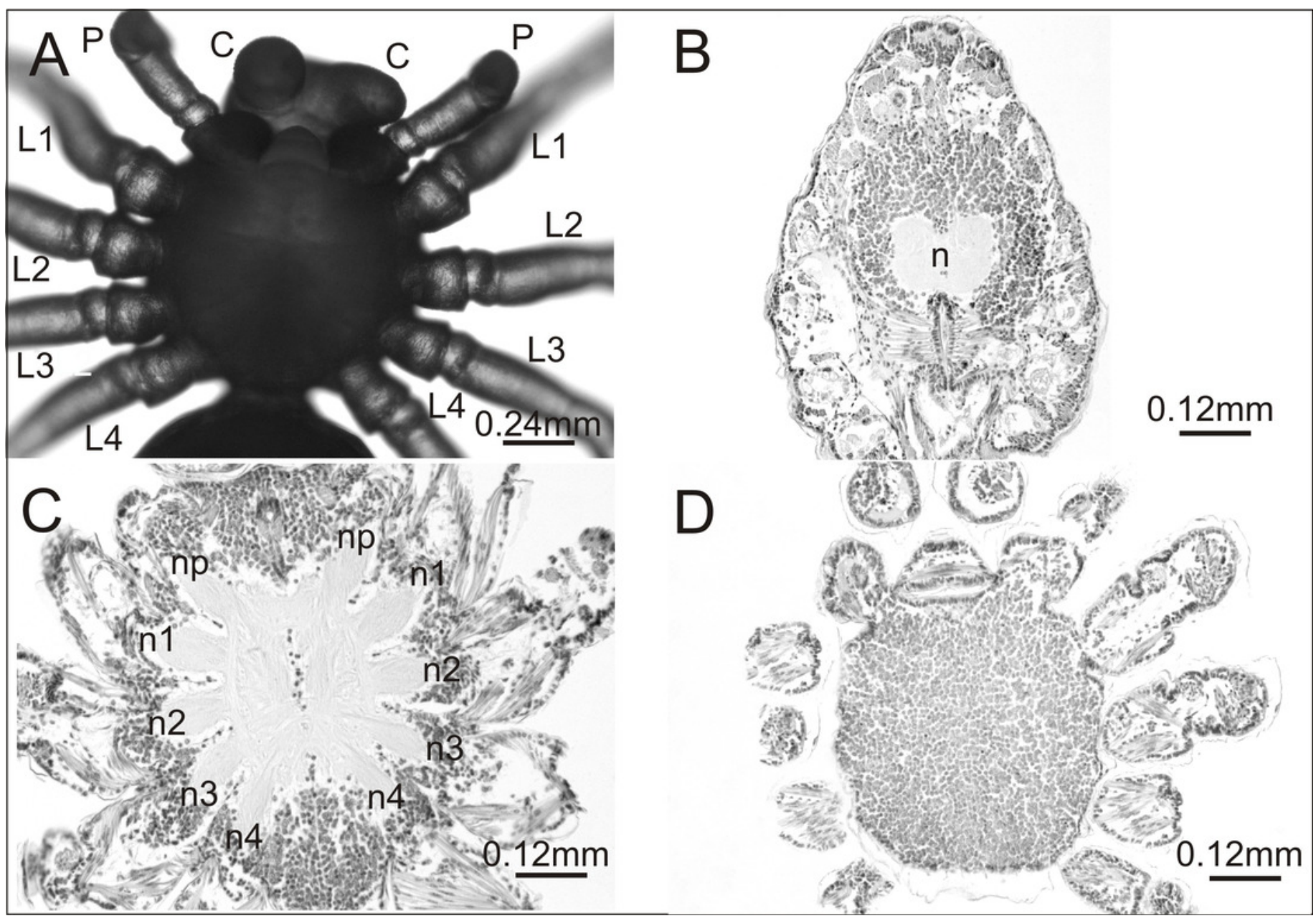




\section{Figure 2}

Eratigena atrica postembryos with teratogenic changes.

A ventral view: postembryo with bilateral oligomely and a protuberance in place of the right chelicera; B dorsal view: postembryo with a protuberance in place of the right pedipalp; C ventral view: postembryo with oligomely of the walking legs, schistomely of the left pedipalp, and a protuberance between the pedipalp and walking leg; D ventral view: postembryo with oligomely of the walking legs on the right side of the prosoma and schistomely of the first left walking leg; E dorsal view: bicephalous postembryo with additional, well-developed walking legs between two heads; $\mathbf{F}$ ventral view: bicephalous postembryo with additional, partially fused walking legs between two heads; A, protuberance; C, chelicera; L, L/L, L1-L4, walking legs; P, pedipalp; white lines indicate the heads of bicephalous postembryos. 


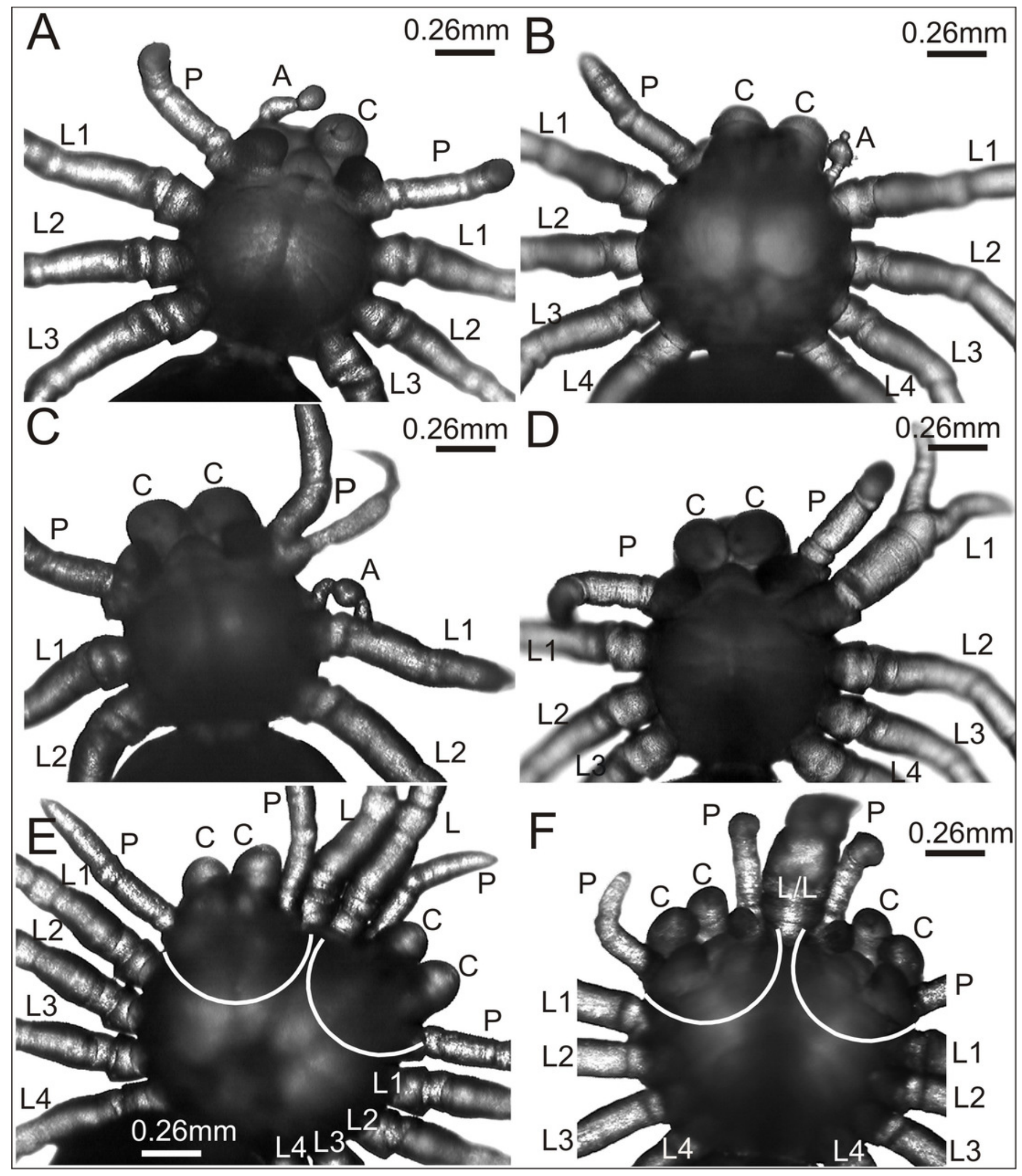




\section{Figure 3}

Eratigena atrica postembryo with complex anomaly.

A dorsal view: a, deformed appendage; L1, L2A/2B, free ends of fused walking legs; P, pedipalps; L1-L4, walking legs; B-D horizontal sections through the prosoma, brain (B) and ventral nerve cord (C, D) (right side abnormal, left side normal): a, fused part of the legs; n1, n2A (C) and n2B (D), neuropils of heterosymelic legs; n1-n4, neuropils of the well-formed walking legs; $n p$, neuropils of pedipalps; $n$, neuropil.

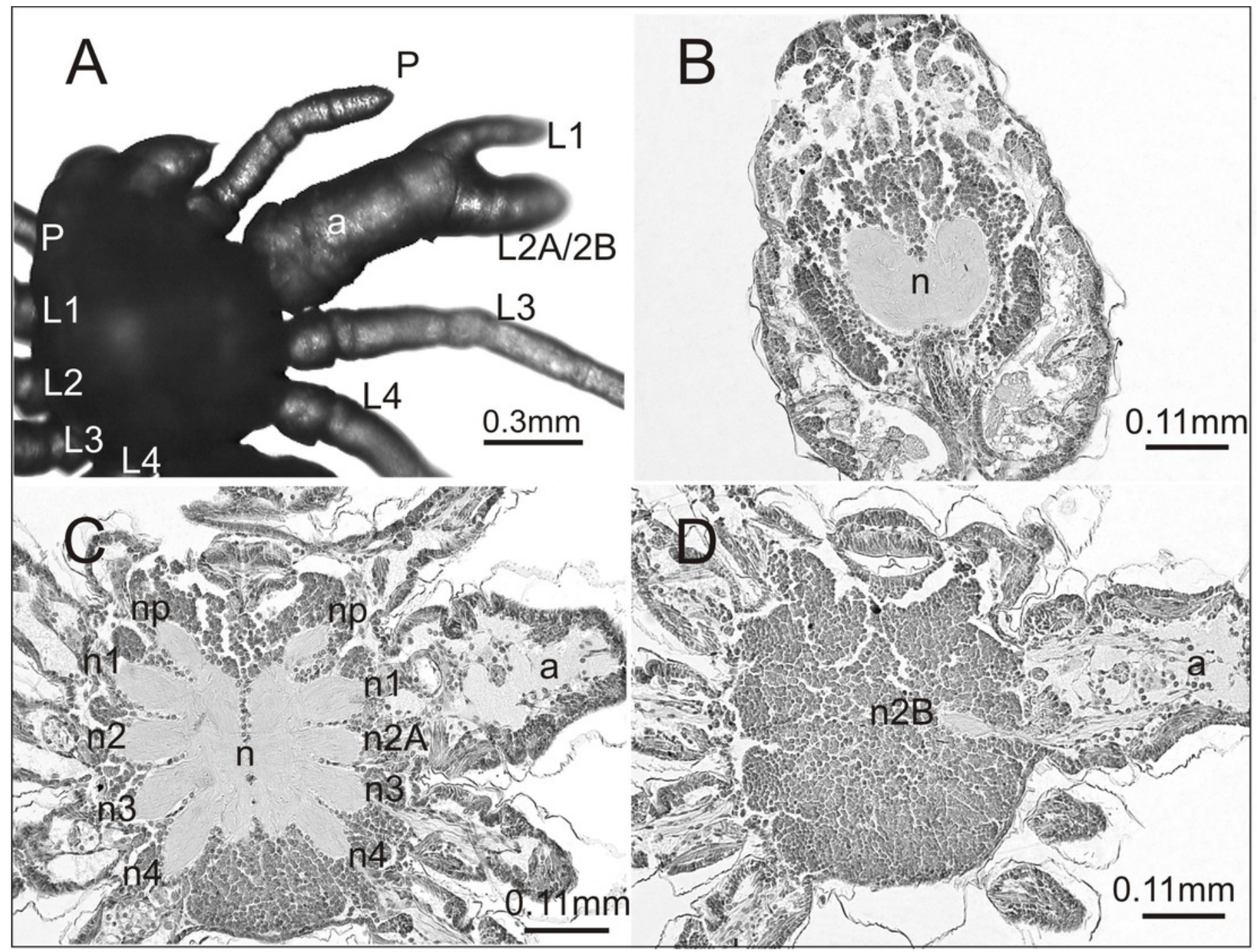

